\title{
Retour amont, sens et expérience grecs du divin
}

\author{
Jean Lauxerois
}

\section{(2) OpenEdition}

\section{Journals}

Édition électronique

URL : https://journals.openedition.org/alter/2037

DOI : $10.4000 /$ alter.2037

ISSN : 2558-7927

\section{Éditeur :}

Association ALTER, Archives Husserl (CNRS-UMR 8547)

\section{Édition imprimée}

Date de publication : 1 novembre 2020

Pagination : 165-181

ISBN : 978-2-9550449-6-4

ISSN : 1249-8947

\section{Référence électronique}

Jean Lauxerois, «Retour amont, sens et expérience grecs du divin », Alter [En ligne], 28 | 2020, mis en ligne le 22 décembre 2020, consulté le 15 octobre 2021. URL : http://journals.openedition.org/alter/ 2037 ; DOI : https://doi.org/10.4000/alter.2037

Ce document a été généré automatiquement le 15 octobre 2021.

Revue Alter 


\title{
Retour amont, sens et expérience grecs du divin
}

\author{
Jean Lauxerois
}

Pour Jean-Jacques Alrivie

1 «Retour amont »- le sens de cette formule, titre d'un ensemble de poèmes de René Char repris dans Le $\mathrm{Nu}$ Perdu ${ }^{1}$, s'éclaire à la lumière du travail de Giacometti, à qui le poète avait demander d'enluminer son recueil. Dans l'eau-forte « L'homme au bord du précipice regardant dans le vide », le trait ardoisé de Giacometti, émergeant d'une opacité noire, vibre au plus près de l'intention poétique de Char : « Retour amont ne signifie pas retour aux sources. Il s'en faut. Mais saillie, retour aux aliments non différés de la source, et à son œil, amont, c'est-à-dire au pire lieu déshérité qui soit $»^{2}$. S'engager dans un tel "retour amont » à propos du sens et de l'expérience grecs du divin ne serait donc pas remonter à ce qui s'entend généralement comme «la source grecque » ou à ce que la philosophie nommerait «commencement ». La poésie le dit autrement : « Revers des sources : pays d'amont, pays sans biens, hôte pelé, je roule ma chance vers vous $»^{3}$.

2 Cette âpreté déshéritée indiquerait le site de l'expérience radicale que les Grecs surtout ceux "d'avant Socrate », dirait Nietzsche - ont faite tout ensemble et du dieu et de l'homme, puisque la profondeur de l'expérience grecque du divin a été d'ouvrir l'homme à sa vérité de "mortel»(brotos). Or cette expérience fut poétique - et notamment tragique - plus que religieuse, dans la mesure où elle excédait, et souvent déboutait, les formes de la religion instituée, qui n'en était ni le cadre ni la mesure.

3 Au demeurant, parler de « religion » des Grecs repose sur un malentendu radical : les Grecs, en effet, ne disposaient dans leur langue d'aucun mot pour dire un tel phénomène; et ce que les Romains furent les premiers à nommer religio relevait d'une relation avec les dieux totalement étrangère à celle des Grecs. Plus encore : le mot « religion » apparaît d'autant plus inapproprié que sa fortune a été liée à son emploi par l'esprit du XVIII et par l'anthropologie du XIX ${ }^{e}$ siècle : il leur fallait rassembler la multiplicité des croyances sous un seul terme, au service de leur dessein qui fut tour à 
tour, ou simultanément, critique, historisant et anthropologique. Or, dans aucun cas, le terme de "religion » n'a été élevé à la dignité d'un concept, ni philosophiquement questionné quant à sa provenance.

4 Situer ainsi le sens que les Grecs avaient du divin, et dans leur sillage, "rouler sa chance » jusqu'au pays d'amont, pour nous qui sommes bien loin vers l'aval, ou plutôt dans le déval, ce serait, en filigrane, tenter une expérience de pensée qui saurait à présent nous mener en amont de toute idée de religion, et du christianisme en particulier. Loin de se réduire à son historicité ni à son archaïcité, l'expérience grecque du divin pourrait bien plutôt apparaître comme un avant-courrier primordial dans la manière à venir de considérer l'homme dans son rapport religieux avec le divin ${ }^{4}$.

\section{Quel est, en amont de toute religion, le sens grec du divin?}

5 Qu'est-ce qu'un dieu pour les Grecs? Pourquoi le rapport avec le divin n'est-il pas pour eux d'abord religieux? Et pourquoi même toute forme statutaire de culte se voit déboutée, notamment dans la recherche tragique du salutaire?

\section{A. Qu'est-ce que le divin?}

6 Le divin, ce sont bien sûr les dieux. Ou des dieux. Comment entendre ce pluriel et ce multiple? Non au sens de ce qu'on entend par « polythéisme ». Il ne s'agit pas de savoir s'il y a beaucoup de dieux ni combien, mais de voir que le divin se déploie sur plusieurs plans - pollakôs, « de manière multiple », comme le dira Aristote de l'être.

7 1. Les deux plans premiers sont ceux dont W.F. Otto ${ }^{5}$ a montré qu'ils correspondaient à deux époques : celle des anciens dieux, qui ont laissé le premier rang aux nouveaux dieux, les Olympiens. Les anciens dieux, comme les Titans, ou les Euménides, sont définis par le terrestre chtonien, le nocturne et la nuit, la mort et le sang, le féminin, l'élémentaire de l'animalité. Ils cèdent la place à ces dieux nouveaux qui apparaissent chez Homère, marqués par le céleste, la lumière du jour, la vie, le masculin - Zeus, Apollon, Hermès, Poséidon, Athéna... Précisons cependant que ces deux plans ne se succèdent pas seulement. D'une part, les nouveaux dieux naissent souvent de la métamorphose de dieux plus anciens - ainsi les Muses, filles du Ciel et de la Terre sous le régime des anciens dieux, deviennent filles de Zeus. D'autre part, les anciens dieux ne disparaissent pas purement et simplement ; ils perdurent au contraire, comme en sousœuvre, lors même que triomphent les Olympiens - ainsi dans l'Orestie, où la justice est l'enjeu d'un conflit entre anciens et nouveaux dieux.

8 2. À ces deux premiers plans, imbriqués, s'en ajoutent deux autres. Dans Das Wort der Antike ${ }^{6}$, W. F. Otto évoque ce qu'il nomme le tournant spirituel du VI ${ }^{e}$ siècle av. J.-C., qui correspond à l'apparition de la tragédie attique et de Dionysos. Avec la tragédie, et tout au long $\mathrm{du} \mathrm{V}^{\mathrm{e}}$ siècle, Dionysos s'impose comme un dieu central à Athènes. Dieu tragique, dieu souffrant, Dionysos n'est évidemment pas un Olympien. Homère le mentionne à peine, le qualifiant simplement de mainomenos ${ }^{7}$, « fou ». Cette mania, cette « folie » va devenir le cœur de la tragédie. Au cœur du déchirement, dans la tension des contraires, Dionysos est le dieu d'une autre rencontre, celle d'une proximité fulgurante dont la violence peut saisir l'homme dans la folie, l'extase, l'excès, l'ivresse, bref dans 
un arrachement qui ouvre sur une expérience d'abîme (chaos). Dionysos est ce dieu inquiétant que l'Athènes du $\mathrm{V}^{\mathrm{e}}$ siècle a pourtant accueilli, acclimaté et honoré ; il est ce dieu sauvage qui bouleverse l'économie du monde réglé par les Olympiens. Au demeurant multiple et multiforme, son mythe même, celui de sa naissance, de sa mort et de sa renaissance, celui de son démembrement et de son remembrement, est l'occasion de déjouer les liens entre toutes les catégories instituées de la vie : il est à la fois enfant, homme et femme; il inquiète le rapport entre l'homme et l'animal, entre la singularité et l'espèce ; il dit aussi la folie maternelle dans la démesure du sein... Ce dieu énigmatique, dont le culte fut aussi cathartique, est un dieu majeur car, comme le dit Otto, c'est grâce à Dionysos tragique que s'est approfondie l'expérience du rapport poétique entre l'homme et le dieu, jusqu'à leur mutuel amont. [Dionysos garde toujours une part d'énigme. Heidegger, l'évoquant à deux reprises au moins, curieusement coupe court. Dans Nietzsche, il interrompt rapidement le développement amorcé sur Dionysos, puis celui sur la question du chaos $^{8}$. Dans Essais et Conférences ${ }^{9}$, une note sur l'Éternel Retour du Même renvoie simplement l'abîme du Dionysos nietzschéen à un abîme plus radical, à la fois impensé et "fermé à la métaphysique » - l'abîme du chaos grec, clef de la pensée d'un négatif qui ne relèverait pas de la métaphysique, écrit Heidegger dans Besinnung ${ }^{10}$.]

9 Nous avons donc déjà trois plans du divin, auxquels il convient d'en ajouter un quatrième - qui deviendra le premier à la fin du monde grec. Ce plan relève des cultes dits à mystères, liés à l'orphisme et au pythagorisme. Platon y fait allusion dans le début de la République, lorsqu'il évoque ces livres de Musée et d'Orphée qui, dit-il, ont été produits en abondance, et auxquels se réfère aussi le Socrate de l'Apologie $e^{11}$. Fondé sur la légende de la descente d'Orphée aux enfers, l'orphisme envisage notamment un tout autre rapport à la mort que celui qu'implique le divin de nos trois premiers plans, dans la mesure où il paraissait assurer à l'initié une manière de triomphe sur la mort. Cette tradition touche à celle des Mystères d'Éleusis, sous l'égide de Déméter. Sans entrer ici dans l'examen savant du lien entre orphisme et cultes d'Éleusis, notons ce qu'en dit Otto dans Die Gestalt und das Sein ${ }^{12}$ : les participants aux cultes éleusiniens étaient conviés à la vision d'un épi de blé, qui ne signifiait sans doute pas le chemin vers l'immortalité, mais l'idée que, malgré la mort d'un individu, la vie continue son cours ("Si le grain ne meurt... »). La question reste ouverte de savoir si ce quatrième plan du divin est profondément grec ou non. Dans les Leçons sur la mythologie, Schelling admet que l'origine des cultes orphiques n'est peut-être pas grecque, mais il convient aussi d'une communauté possible entre monde grec et monde oriental. Tout en les restituant à la grandeur initiale de leur mythe, W. F. Otto, lui, affirme qu'au regard de l'apogée grecque du divin les cultes orphiques demeurent comme un «à côté ». Il n'en reste pas moins que, pour n'être pas central dans la Grèce classique, ce rapport au divin connaîtra une destinée importante dans le monde hellénique et méditerranéen ; joints à d'autres cultes, notamment romains, ces cultes à mystères accréditeront peu à peu l'idée d'immortalité.

10 Nous avons donc désormais quatre plans différents : celui des anciens dieux, celui des nouveaux dieux Olympiens, celui de Dionysos et celui des cultes à Mystères. Serait-ce tout? Non. Et c'est ce qui rend si riche et si complexe le sens que les Grecs avaient du divin, une fois dépassée la conception réductrice voire simpliste du prétendu "polythéisme ». 
11 3. Trois plans doivent encore s'envisager: le divin peut être encore primordial, immémorial et démonique.

12 Prenons ici référence à Homère. Lors même qu'elle consacre l'entrée en scène des grands dieux Olympiens, l'Iliade reste entièrement ouverte à la présence d'autres dieux. Les dieux primordiaux relèvent de la puissance des éléments, et sont généralement invoqués pour garantir un serment. Au chant III, par exemple, Agamemnon peut ainsi prier : «Zeus, et toi, Soleil, qui vois tout et entends tout, et vous Fleuves et Terre, et vous enfin qui, dans les abîmes souterrains, punissez après leur mort les hommes qui ont été parjures, soyez-nous témoins et veillez à la foi de nos serments ${ }^{13}$. Mais Homère donne plus de place encore aux divinités immémoriales qui donnent figure à la nécessité et règlent le jeu de la vie et de la mort, telles Moira, Anankè, Tukhè, Atè, Éris... Moira est la plus imposante d'entre elles : liée à la mort (souvent nommée la Kèr, déesse de la «mort noire»), elle est à la fois l'Échéance et le Lot destinal. Les dieux, Zeus et Héra en tête, sont eux-mêmes soumis à « la puissante Moira ». Héra peut dire à Achille : "Ce n'est pas nous qui causerons ta mort, mais un grand dieu, la puissante Moira » ${ }^{14}$. Jean Beaufret : «Le dieu n'est jamais, dans le muthos grec, le dernier mot, c'est-à-dire l'absolu de la question, mais, au-delà du divin, le destin auquel le dieu lui-même demeure soumis. En d'autres termes, il y a une plus haute énigme que le divin ${ }^{15}$.

Nuançons cependant : nous ne dirions pas «le divin », mais «le dieu», car le plan de Moira est aussi un plan divin, dont le dieu, notamment olympien, n'est que l'une des figures possibles. Plans des divinités primordiales et des divinités immémoriales, donc, mais aussi et enfin plan du démonique. Ce plan reste le plus énigmatique, car le daimôn qui traverse le monde grec, d'Homère aux Tragiques, des Tragiques à Socrate, est un dieu innommé, parfois innommable. Certes les dieux, tels Zeus, Apollon ou les Euménides, peuvent être dits "démoniques", mais daimôn nomme aussi un plan tout autre, insituable, de la relation entre l'humain et le divin. Sa puissance est ambivalente : elle peut être maléfique autant que tutélaire - en ce sens, un Grec ne manque jamais de souhaiter à ses proches, à ses amis, comme à soi, la chance d'un bon daimôn.

\section{B. Pourquoi la relation avec le divin n'est-elle pas religion ?}

14 1. Dans un texte de 1951, intitulé « Rom und Griechenland $»^{16}$ (« Rome et le Grèce »), W.

F. Otto précise que la question la plus épineuse est de savoir comment nommer la Frömmigkeit des Grecs, leur « piété ». Selon lui, il est fort difficile de lui trouver un nom, et c'est seulement parce qu'il faut bien la différencier qu'il choisit, faute de mieux, de l'appeler « une religiosité profane ». L'oxymore vient donc parer au défaut du mot, qui relève moins d'une lacune du lexique qu'il ne trahit une défaillance de la pensée, impuissante à nommer ce qui demeure pourtant son amont, l'entité grecque du divin. La raison en tient, entre autres, au passage du monde grec au monde romain, de la langue grecque à la langue latine, laquelle nous a donné les mots de "religion », « foi », " croyance ", " confession », dont l'histoire a ensuite accrédité l'évidence. Or, comme Otto le précise, la religion romaine est bien au plus loin de l'expérience grecque du divin.

Relevons trois aspects majeurs de la religio : 1/le dieu des Romains est avant tout numen, une "volonté » qui implique qu'on prenne garde à elle jusque dans l'attention superstitieuse aux signes (ainsi les jours fastes et néfastes, la façon dont on franchit un 
seuil...). En ce sens, comme le précise Cicéron, le mot religio est étymologiquement rattaché au verbe relegere (et non, comme le dit Lactance, au verbe religare, "relier »). W. F. Otto, comme Benveniste ${ }^{17}$, donne raison à Cicéron : la religio, au sens de re-legere, c'est la prise en garde scrupuleuse de la volonté divine (positive ou négative), et au nom de ce scrupule respectueux elle est la manière dont l'homme sait se montrer juste envers les dieux. 2/Nous tenons là le deuxième aspect de la religion romaine : le dieu romain relève de la sphère proprement romaine du jus, donc du droit: comme le dit encore Cicéron de la piété envers les dieux, la religion est une " justice " à l'égard des dieux. 3/Enfin, cette dominante juridique fonde la séparation toute romaine entre le droit privé et le droit public : le droit sacré devient partie du droit public et la religion devient alors l'affaire de l'État - voire une affaire d'État.

Que ce soit donc au sens romain ou au sens généraliste de l'anthropologie moderne, les Grecs anciens ne connaissent pas à proprement parler de "religion»- eux dont la langue, nous le disions, ne dispose d'aucun terme pour nommer un tel phénomène. Ils n'ont pas non plus de «croyance». W. F. Otto : «Il n'y a pas de "croyance“ pour les Grecs. Qui utilise ce terme a bien peu compris le savoir grec du divin ${ }^{18}$. Certes, Otto lui-même tombe ici ou là dans le piège du lexique ordinaire - son livre Theophania est sous-titré «L'esprit de la religion grecque ancienne ». Mais l'embarras vient de ce qu'il est obligé, contre les détracteurs du paganisme (chrétiens ou rationalistes), de défendre l'idée que les Grecs avaient une haute compréhension du divin autant que du sacré. Et malgré telle petite inadvertance Walter F. Otto est bien le seul qui permette de penser le dieu grec en sa différence. C'est là ce qui peut l'opposer à Rudolph Otto, dont l'ouvrage, Le Sacré1 ${ }^{19}$, a beaucoup contribué à populariser la notion de "sacré » en l'instituant comme catégorie universelle. Si Rudolph Otto est beaucoup plus connu que Walter Friedrich Otto, il n'en demeure pas moins que son parti souffre d'une généralisation anthropologique, qui méconnaît la dimension spécifiquement grecque d'un phénomène dont l'origine est d'abord romaine. Quand nous disons "sacré », en effet, nous parlons latin, non grec. Le sacer des Romains, c'est le "séparé ", qui présuppose la séparation du profane et du religieux. Le sacré séparé, c'est notamment le tabou, l'être impur, intouchable, qui peut d'ailleurs désigner aussi bien le meurtrier que la victime, et tout autant l'espace qu'il est interdit de franchir, parce qu'il a été marqué par le sang versé. Or, même s'ils ne méconnaissent pas un tel phénomène, rien n'est plus étranger aux Grecs qu'une telle séparation, car le sacré serait plutôt pour eux celui d'une sainteté, au sens où Baudelaire parle du "saint foyer des rayons primitifs $»^{20}$ : le sacré est ce qui rayonne à même le kosmos du monde, à partir d'un foyer secret, qui, sans se montrer lui-même, ouvre à l'articulation ou à l'unisson du divin et de l'humain.

17 2. Donc, mieux encore que de "religiosité profane", parlons avec W. F. Otto de Frommigkeit, de "piété » (même si le mot est lui aussi de provenance latine) : en grec, eusèbeia. À condition de préciser que cette piété n'est l'affaire d'aucune révélation, d'aucun article de foi, d'aucun texte canonique, ni même d'aucune mythologie puisque le mythe grec n'a jamais relevé de ce qui s'est tardivement constitué comme mythologie, dont les Modernes ont fait ensuite une catégorie anthropologique. Et à condition d'ajouter avec Otto que, mieux encore que de piété, il faudrait parler, avec Hölderlin, de « proximité du divin »" .

18 Cette piété est de l'ordre du mythe et du culte à la fois. Le mot «mythe » parle grec, puisque le muthos est la parole d'autorité qui dit ce qui est pour avoir toujours été - 
tandis que le logos dit la parole qui médite et recueille. Le mythe est poétique et tragique, il dit une expérience irréductible du rapport avec le divin. Le mythe est irremplaçable, car «supprimer le mythe et vouloir le remplacer par une prétendue expérience pure du dieu, c'est renoncer à la proximité du dieu $»^{22}$. Donc, loin de toute généralisation réductrice, l'approche de la dimension proprement grecque du mythe le récuse comme mode de pensée archaïque. D'ailleurs, il n'y a pas de "pensée mythique » en général. Le mythos est grec : il est toujours l'expérience d'une rencontre particulière entre l'homme et le dieu, laquelle advient dans le monde. Mais mythe et culte ne doivent pas être dissociés ${ }^{23}$ : leur différence est que le culte adviendrait plutôt comme geste, tandis que le mythe serait parole. Dans le culte, l'homme s'élève jusqu'au divin en agissant en communion avec lui, tandis que, dans le mythe, c'est le divin qui s'abaisse jusqu'à l'homme auquel il emprunte, dit Otto, une "figure», Gestalt (terme déjà présent chez Winckelmann). Le dieu devient ainsi «la figure de toutes les figures $»^{24}$. Les figures agissent ainsi en profondeur et donnent à sentir l'être des choses, qu'elles délimitent dans leur unité, dans leur plénitude et dans leur beauté. La délimitation qu'est la figure est à la fois exclusion et inclusion, ouverture et clôture : la limite est donc un tout. Et figure le tout. Le mythe fait et donne figure. Il englobe alors le culte, dans la mesure où la figure est déjà manifeste dans le corps dressé de l'extase, de la prière, de la danse, puis dans l'édification - de la statue, du temple, etc. - jusqu'à s'accomplir enfin dans la parole et dans la musique.

19 3. Néanmoins, cette piété grecque ne serait pas sans un ultime élément, ultime mais premier à vrai dire : le lien avec les morts. Non la mort, mais bien les morts. La piété envers les morts est la clé du rapport grec entre l'humain et le divin - depuis Homère jusqu'à Sophocle. Le cœur de l'Odyssée, par exemple, c'est la descente d'Ulysse aux Enfers. Cette descente ordonnée par Circé répond à un très rigoureux rituel que la magicienne détaille à Ulysse, et qu'il suivra avec scrupule pour accéder à l'âme des défunts. Ce voyage d'Ulysse est le moment majeur de son aventure : c'est dans cette piété montrée envers les morts que se découvre, au cœur de l'expérience, non seulement l'âme des morts, mais la vérité des mortels que sont les vivants. En ce sens, même s'ils l'ont en horreur, la mort n'est rien de morbide pour les Grecs : la question n'est pas pour eux celle de « savoir mourir » (thème de la « sagesse » romaine), mais de savoir vivre en mortel.

Et c'est aussi la leçon d'Antigone. Lorsque Créon ironise en disant Antigone qu'elle doit se réjouir de son châtiment puisqu'elle n'a de piété que pour Hadès et pour ceux qui sont chez Hadès (vers 777-780), le chœur répond en jouant sur le lien entre sebein et eusebein : «Révérer les morts (sebein), oui, c'est cela la piété (eusebein)» (vers 872). Rien de plus divin que le lien d'égards qui relie les vivants et les morts. Et c'est cette piété qu'Antigone veut et pense incarner : «Je ne suis que piété, et c'est moi qu'on accuse d'impiété!» (vers 924). Au-delà de toute forme statutaire de respect, une telle piété n'est même pas révérence envers les dieux : elle revendique bien plutôt le rapport des hommes avec leur amont, c'est-à-dire avec leur être mortel, qui apparaît à la lumière de cette communauté des morts et des vivants dont Antigone affirme la primauté. Là le cultuel ne saurait pas suffire. Les Grecs le savaient, qui ont voulu remonter jusqu'à l'amont de toute forme de culte - lequel, au demeurant, n'était déjà pas seulement «religion ». 


\section{Du statutaire au salutaire}

21 Le sens grec du divin ouvre en effet à une expérience de la limite et de la métamorphose, jamais seulement instituée, toujours à l'œuvre.

1. Cette expérience est toujours un événement, lors duquel le dieu et l'homme se rencontrent. Otto: «[...] quelque chose advient [...]» ${ }^{25}$. Et cet événement, ou avènement, doit s'entendre comme un kairos, le surgissement d'un instant décisif où le dieu rencontre l'homme comme sa cible. Otto écrit à propos d'Achille : « Dans l'instant, l'homme et le dieu sont si proches que la différence entre eux est abolie. C'est là l'expérience que les Grecs anciens ont faite du divin, et que ne saurait dévaluer le dogmatisme d'aucun savoir prétendument supérieur $»^{26}$.

23 Cette dimension est fulguration, présente et intemporelle à la fois. Et dans ce face-àface de la rencontre, comme nous l'avons dit du mythe, advient une reconnaissance mutuelle dans laquelle chacun prend « figure », la figure étant la forme accomplie de ce qui se déploie selon sa nécessité intime et à la mesure du monde. Dans leur rencontre, l'homme et le dieu adviennent donc à leur délimitation réciproque.

24 2. Une telle délimitation concerne en priorité l'amont de l'être de l'homme, que la rencontre délimite comme limite: le sens du divin est de faire apparaître l'homme (anthropos) comme le «mortel » (brotos). La rencontre le délimite ainsi en sa mortalité. Pensons ici au célèbre fragment 62 d'Héraclite :

Immortels-mortels mortels-immortels

Vivant de la mort des uns

Déjà morts de la vie des autres.

Hommes et dieux se dé-finissent et vivent de leur rapport mutuel, les dieux immortels au miroir de la mort des mortels, les mortels existant au miroir de l'immortalité des dieux. Mais les hommes ne vivent pas! Ils sont tethneotes - le grec utilise ici le temps dit du parfait - c'est-à-dire "déjà morts ». Au fond de toute vie humaine, il y a déjà la mort : tel est la vérité de l'être mortel, tel est notre amont, "pays déshérité ». L'être de l'homme est un défaut d'être, le mortel est en défaut d'être, et rien n'est plus divin. À cet égard, la célèbre inscription du temple d'Apollon, gnôthi seauton, n'était pas un appel à l'introspection, mais à la reconnaissance de ce défaut: "Sache que tu n'es pas un dieu ! " La présence du dieu Olympien tenait dans cette injonction : Sache ce que tu n'es pas! Apprends à te connaître comme n'étant pas! Et les Grecs sont allés plus loin encore pour approfondir l'essence du mortel en son défaut d'être.

3. Tel est l'horizon du tragique, car la tragédie attique est le lieu poétique de l'expérience inouïe, qui bouleverse les limites établies, grâce auxquelles l'humain et le divin étaient à la fois liés et séparés. La tragédie fait passer le mortel d'une dimension statutaire à une dimension salutaire (distinction que nous reprenons à l'esprit des Remarques sur CEdipe et Antigone de Hölderlin). Le statutaire relève du culte en son assise rituelle. Statutaire est aussi la ligne de partage que le mythos assigne poétiquement aux dieux et aux mortels. Or ce partage n'est pas définitivement institué, il est toujours à rejouer, à l'œuvre, en amont de tout existant. Et telle est la destination de la tragédie, notamment dans l'expérience de la folie.

Grâce à la tragédie, la folie est l'expérience du divin la plus radicale que le monde grec nous ait donnée, dans la mesure où il a osé aller au-delà de lui-même, en déroutant, en déboutant et le culte statutaire et le mythe, pour trouver la voie du salutaire. Ce salut (Heil) du salutaire n'est pas réductible au sacré (heilig) de la romanité ni non plus à la 
catharsis dont Aristote ouvrira la longue histoire. Le salutaire tragique est précisément "l'amont », « l'œil » qu'évoque René Char, «le pire lieu déshérité qui soit ». Le Chœur d'Antigone illustre le sens de la présence de Dionysos dans la tragédie. Le premier chœur fait appel au Dionysos cultuel pour célébrer la fin de la guerre et l'unité retrouvée. Mais les chœurs suivants sont d'un autre ordre : le deuxième évoque l'homme sous le signe du deinon, de "l'effrayant », en ouvrant à la dimension de la scission et du déchirement, et en signant l'ouverture du temps, centre du troisième chœur ; dans le quatrième, Zeus se temporalise à son tour, selon un retournement tragique du mythe de Danaé - comme l'a vu profondément Hölderlin. ${ }^{27}$ Le temps apparaît ainsi comme le nom secret du plan profond du divin. Aussi bien, l'avant-dernier chœur fait réapparaître les dieux de la destruction et de la métamorphose, qui règlent le jeu du monde : Éros, puis Dionysos, que le dernier chœur célèbre pour en appeler in fine à l'accomplissement d'une nouvelle présence. Dans le retournement opéré par l'insurrection tragique advient la métamorphose : Dionysos, dieu du théâtre et de la folie, dieu "aux noms multiples ", qualifié de "tout commun », figure le lien d'une communauté neuve, qui n'est plus seulement cultuelle, puisqu'elle est désormais l'affaire poétique du théâtre. Dionysos est le dieu de la célébration régulatrice de la vie et de la mort, dont la poésie tragique, non le seul culte, approfondit la nécessité et la grandeur.

\section{La manifestation du divin et la métamorphose du mortel}

Il n'y a donc de mortel qu'à la mesure de la phénoménalité des dieux en leur manifestation. Alkinoos le dit à Ulysse: "Les dieux se manifestent de manière éclatante, phainontai enargeis ${ }^{28}$. Ce phénomène a pu être nommé en grec theophania, terme qui associe le theos, "dieu » et le substantif issu du verbe phainomai, " apparaître, se manifester ». Au pluriel, le mot désigne chez Hérodote la fête où l'on exposait les statues des dieux. Il est repris dans la patristique, chez Grégoire de Nysse par exemple, pour nommer l'apparition de Dieu à Moïse - et il est clair qu'une telle nomination n'est possible qu'à partir d'une pensée grecque de la theophania.

1. Cette theophania, W. F. Otto la reprend à son compte, pour dire l'expérience du divin comme rencontre de ce qui est et qui se manifeste. C'est en ce sens qu'il peut écrire qu'il n'y a pas de croyance pour les Grecs, puisque la rencontre vit au foyer de ce qui est présence. Otto : «Les dieux sont et sont manifestes (offenbar) »29. Et il peut ainsi ajouter que la manifestation du divin est la clef des tragédies d'Eschyle. Manifestation? Otto choisit parfois Offenbarung, parfois Manifestierung ${ }^{30}$, voire Manifestation. Il s'inscrit là dans une tradition de la langue allemande, ouverte par Winckelmann, qui parle d'Offenbarung devant la statue d'Apollon; mais Goethe est le premier à utiliser Manifestation, lequel écrit par exemple : Das Schöne ist eine Manifestation..., « le beau est une manifestation... $»^{31}$. Curieusement, la pensée ne s'est pas vraiment intéressée à ce terme, au point que le flou, philosophique et théologique, a été trop entretenu, entre les termes "révélation ", " apparition ", " manifestation ", voire "phénomène »... Le mot «manifestation », dont la provenance, sans doute théologique, demeure obscure, apparait dans la langue philosophique chez Hegel, qui l'emploie le plus souvent au sens de "phénomène ». Goethe, lui, est plus attentif à la précision, lorsqu'il écrit avec clarté : « Le vrai est semblable à Dieu. Il n'apparaît (erscheint) pas immédiatement ; nous devons le deviner à partir de ses manifestations (Manifestationen) $\rrbracket^{32}$. 
2. Au sens où Goethe et Otto emploient le terme, quels seraient alors les modes concrets de la «manifestation » de la présence du dieu grec en son éclat? Sans avoir la toute puissance, les dieux sont, dit Homère, les "toujours étants", ou encore "ceux qui vivent dans la joie et la légèreté » : ils se manifestent ainsi dans la beauté du monde (kosmos), là où le dieu et l'hommes s'ouvrent mutuellement l'un à l'autre, en un ajointement secret qui unit les contraires. Mais, ajoute Otto, le dieu grec se manifeste aussi jusque dans "ce qui anime l'homme au plus intime de lui-même ». Dès lors, rien de ce qui fait vivre et agir les hommes n'est étranger à la dimension du divin. Au sens où Télémaque, partant à la recherche de son père, dit à l'inquiète Euryclée : « Rassuretoi, nourrice, car, bien sûr, sans un dieu, pas de pareille décision $»^{33}$. Les dieux peuvent toutefois être très ambivalents dans leur proximité envers les hommes. La présence divine peut être inattendue: indifférente, tutélaire ou hostile. Hermès, Athéna protègent Ulysse, mais, comme le dit aussi Hermès, « un dieu est toujours capable de mauvais desseins $»^{34}$. C'est bien ce qui, entre autre, contribue à dessiner la limite qui sépare, dans leur rencontre même, mortels et immortels. Enfin, "les dieux ne se manifestent pas à tous de manière éclatante (enargeis) $»^{35}$. Ulysse peut discerner la présence d'Athéna, mais Télémaque ne la voit pas. Autrement dit, toute manifestation divine appartient à l'alètheia grecque, toujours habitée par le nuage de la lèthè, qui, comme le dit Pindare ${ }^{36}$, peut voiler, obscurcir ou dissimuler l'éclat même du vrai, tant c'est toujours un retrait, une réserve, qui porte la possibilité de toute manifestation.

313 . Aussi bien, pour dire ce plan de toute manifestation possible, nous retiendrons le mot grec de phasma ${ }^{37}$, qui peut permettre de situer l'amont de tout apparaître, de la phénoménalité et de la theophania du dieu grec. D'emploi rare, le terme phasma apparaît dans Phèdre (250bc), à propos du beau : «Intègres, simples, immobiles et bienheureuses sont les manifestations (phasmata) auxquelles nous avons été silencieusement initiés dans l'éclat d'une pure lumière, car nous étions purs [...] ».

De ce phasma, étrangement laissé pour compte par la tradition du commentaire philosophique de Platon, retenons seulement que la brillance de ce que Socrate nomme un peu plus loin « ce qui se manifeste avec le plus d'éclat (ekphanestaton) et qui suscite le plus d'amour (erasmiôtaton)» s'adresse désormais à une aisthesis qu'on peut dire « extatique » (dirait Baudelaire). Le beau est l'affaire d'un sentir radical, qui n'est pas de l'ordre du seul visible, mais d'une ouverture qui perce (existanai) jusqu'à cette plénitude qualifiée d'« intègre, pure, immobile et bienheureuse ».

Précédant Platon, Eschyle est néanmoins le premier à employer le mot phasma, qu'on trouve au pluriel, à deux reprises, dans Agamemnon (vers 144-5 et 274). Le génie d'Eschyle s'entend à faire vivre d'emblée deux plans qui définissent le jeu duel dans lequel s'inscrivent les mortels. Ainsi le veilleur, qui attend le signe de la victoire, est dans l'entre-deux, entre ciel et terre, entre hier et demain. Il attend le symbolon, le signe avant-coureur - ce qui tient ensemble, dans l'espace et le temps, les deux éléments d'une réalité. Or voici que l'apparition du symbolon, qui devait apporter la joie, vient inquiéter au contraire le plan de la réalité première. Avec les rappels du Coryphée, puis du Chœur, tout s'ouvre à la dimension de Chronos, le temps, et de Moira, le partage destinal; le devin Calchas fait apparaitre la dimension duelle du présent et de l'avenir, qui dédouble le sens des choses, légitimant cette présence du symbolon et des phasmata: "Tant en effet les signes des oiseaux demandent à être accomplis, qu'ils soient des manifestations (phasmata) favorables ou fâcheuses $»^{38}$. Au début de la deuxième partie de la tragédie, dans le passage de la nuit à l'aurore, le 
Coryphée ne sait plus qui croire; le mot phasmata est alors repris, en liaison avec les rêves $^{39}$. Dans cette puissante tragédie, la manifestation du divin est étonnante, car elle est habitée par le jeu des plans multiples que nous avons évoqués: celui de l'immémorial, celui du divin archaïque et souterrain, en conflit avec les dieux nouveaux, celui d'un daimôn innommé, enfin celui d'Athéna, qui vient ouvrir la possibilité régulatrice d'un ordre nouveau à Athènes. La folie est l'épreuve de ces différences de plan, et Dikè, « la justice », en est l'enjeu. Ce qui se manifeste, dans le jeu des phasmata et du symbolon, ouvre au discord tragique, dont l'expérience, par la folie, mènent les mortels jusqu'à l'épreuve de ce qui met en question l'existence de leur communauté.

4. La manifestation du divin est donc, pour reprendre un terme majeur de la pensée d'Aristote, une energeia - avec laquelle consonne l'adjectif enarges, "éclatant de présence ", qui qualifie la manière dont les dieux se montrent aux hommes. Leur energeia, c'est bien l'éclat de leur présence, entendu comme accomplissement et comme métamorphose. La présence transfiguratrice du divin n'est donc pas une essence, mais une plénitude, à l'œuvre comme poièsis - si le verbe poiein ne signifie ni faire ni créer, mais "faire apparaître à la présence", au sens où Socrate l'emploie encore ${ }^{40}$. Le sens grec du divin serait ainsi la poéticitét ${ }^{41}$. Dans l'energeia résonne l'ergon, l'œuvre, la mise en œuvre. Le poète voit les choses à la manière d'un dieu, dont le regard donne à l'homme de se transfigurer en devenant "égal à un dieu " (Télémaque) ou " comme un dieu " " (Ulysse). Et tout élément du kosmos et de la physis peut être convoqué à devenir le visage d'une autre figure du monde: l'apparaître est un comparaître (paraître ensemble), source de toute comparaison.

Le phasma de la manifestation est le plan inapparent qui porte toute possibilité d'apparaître, il est l'energeia qui mène chaque chose à son éclosion et à sa plénitude dans la beauté du kosmos. L'apparaître et le disparaître sont un phénomène divin, comme Athéna ou Hermès surgissent dans l'espace, ou prennent leur envol. Et Athéna peut conférer à Ulysse ou à Pénélope cette aura qui leur donne à leur tour la grâce de l'apparaître. Devant la métamorphose de son père, Télémaque ne peut s'y résoudre: pareille métamorphose de l'apparaître du mendiant ne peut être que celle d'un dieu! C'est pourquoi Athéna peut être comparée à une artiste à l'œuvre, qui fait surgir la beautét2 : elle œuvre, dit Homère, comme l'artiste qui est doté d'une technè, et qui mène à l'exécution d'œuvres irradiant la grâce. Ainsi le poète est bien tel un dieu: il transforme, transfigure et agrandit ce qu'il place dans la lumière de l'apparaître, suscitant l'admiration étonnée devant pareille fulgurance.

Sans pouvoir en dire ici davantage, précisons simplement que l'ex-

périence grecque du divin ne s'arrête pas là. Si le divin permet au mortel d'accéder à sa "constitution ordinaire» (Rimbaud), si le libre jeu de la métamorphose permet au mortel d'advenir à soi, en étant " comme un dieu ", l'homme doit alors d'autant mieux apprendre l'exercice de la limite pour devenir mortel. C'est le sens des deux mots majeurs que sont aidôs «la vergogne » et philia, «l'amicalité » - omniprésents chez Homère, Hésiode, Pindare, Eschyle, Sophocle... La "vergogne", c'est prendre en compte et prendre en garde la présence du dieu ; et c'est par elle que passe la reconnaissance de notre être mortel. Émanant de l'exigence de la mesure, au regard de la différence qui sépare, tout en les reliant, les mortels et les immortels, la vergogne forme le principe d'un savoir vivre, que la philia doit accomplir. À hauteur d'homme, en effet, le sens de la limite s'éprouve dans le phénomène de «l'amicalité ", qui nomme le 
lien de "communauté" (dira Aristote) s'imposant à tous les vivants. Aidôs et philia participent donc pleinement de cette dimension décisive de l'amont vers laquelle la pensée poétique des Grecs n'a cessé de remonter pour en dire la grandeur divine, et pour former un monde habitable.

Est-il possible d'opérer aujourd'hui un tel "retour amont »? Sommes-nous encore en mesure de nous ouvrir au lieu déshérité de notre mortelle divinité ? Sommes-nous capables d'une telle métamorphose, en un temps où il n'y a pas d'entente de la multiplicité des plans du divin, et où ce qu'on appelle « monothéisme » réduit souvent la plénitude de Dieu même? Il faudrait préalablement sortir de la confusion qu'entretiennent les vaticinations à la Malraux nous annonçant un siècle prétendument " religieux », sans que jamais l'on n'interroge le sens de ce terme, ni la différence entre religion, foi, croyance, piété, sens du divin... Il faudrait aussi échapper une bonne fois à la vision platement historiciste qui, au nom d'un progrès ou à tout le moins d'une évolution supposée, renvoie implicitement l'immémorial à l'archaïsme primaire.

Pouvons-nous entendre aujourd'hui ce que seraient, au pluriel, « les dieux »? Au sens où René Char écrit :

Dieux aujourd'hui sans fonction, sans tribu, quel principe nous fait vos captifs? Vous avez cessé de nous protéger et nous nous sommes approchés de vous, vous avez dépensé votre chaleur, et notre cœur bat dans votre retranchement, vous êtes devenus silencieux, nous vous entourons de paroles d'océans ${ }^{43}$.

Ces lignes éclairent notre rapport avec le divin à présent, en le situant comme expérience du délaissement. Char peut dire encore que nous sommes dans «la faille géante de l'abandon du divin $»^{44}-$ au sens où ce n'est pas nous qui abandonnons le divin, mais bien lui qui nous abandonne. Que le divin puisse abandonner les hommes, voilà qui pouvait parler à l'écoutant des tragédies de Sophocle, mais qui a largement de quoi surprendre l'homme contemporain. Et que, dans pareil abandon, un poète du $\mathrm{XX}^{\mathrm{e}}$ siècle puisse nommer les dieux, et même s'adresser à eux, voilà qui a de quoi dérouter plus encore. Or Char dit bien «les dieux», ajoutant que, dans le retrait du « retranchement » qui est le leur, dans le délaissement où ils nous laissent, dans la déshérence où ils sont eux-mêmes ("Dieux aujourd'hui sans fonction ni tribu... »), quelque chose pourtant nous retient et nous relie à eux, sans que soit nommé le « principe » qui fait de nous leurs « captifs ».

Dans ce délaissement, nous avons aujourd'hui à affronter l'athéisme, à nous situer face à cette "mort de Dieu» dont le discours contemporain emprunte la formule à Nietzsche, tout en confondant trop souvent " mort de Dieu », "absence de Dieu », « retrait du divin », «monde sans Dieu » ... Que les dieux puissent se retirer, que Dieu lui-même puisse nous abandonner, ne signifie pas qu'ils soient morts, ni qu'ils soient seulement absents, ni non plus qu'ils «n'existent pas ». Au sens moderne, Hölderlin fut le premier à nommer le "défaut de dieu » (Gottesfehl) dans le sillage duquel s'est inscrit Heidegger pour en développer la pensée et la profondeur. Sous ce signe, rappelons simplement cet extrait de son Nietzsche:

La disparition de tout salutaire maintient reclus l'espace même du sacré. La réclusion du sacré obscurcit la lumière de tout rapport possible à la Déité. Cet obscurcissement confirme et abrite en lui le défaut de dieu. Un tel défaut, dans l'ombre qu'il répand, laisse tout l'étant se dresser dans l'étrange. [...] L'étrangeté de l'étant comme tel met au jour le dépaysement de l'homme de l'histoire au milieu de l'étant dans sa totalité. Le lieu même où pouvoir habiter au milieu de l'étant comme 
tel paraît anéanti aussi longtemps que l'être se refuse en tant qu'ouverture d'un séjour ${ }^{45}$.

41 Avant d'avoir un jour la possibilité d'affronter cette pensée décisive, le christianisme lui-même aurait à se confronter avec ce qu'a été et demeure son amont. Sans doute lui faudrait-il poser la difficile question de son rapport avec la langue grecque - Nietzsche : "C'est un raffinement que Dieu, ayant voulu se faire écrivain, ait appris le grec, et qu'il ne l'ait pas mieux appris $»^{46}$. Sans doute aussi la question de la mort, c'est-à-dire celle du rapport entre le mortel grec et l'immortalité, entre la mort et la vie éternelle, a ouvert, via le platonisme, un abîme entre le monde grec et l'Occident. Néanmoins le christianisme devra un jour " rouler sa chance " jusqu'à son amont. Il devra mesurer en quoi il a été et demeure profondément habité par le sens grec du divin, qui serait, comme en arrière-plan, le présent, l'intemporel ou le perpétuel de ce que, faute de mieux, nous continuons à nommer aujourd'hui « religion ».

\section{NOTES}

1. R. Char, Euvres complètes, La Pléiade, Paris, Gallimard, 1983, p. 419 sq.

2. Op. cit., «Bandeau de Retour amont ", p. 656.

3. Op.cit., Retour amont, p. 433.

4. Cette présentation s'inscrit dans la ligne de nos ouvrages La Beauté des mortels, Paris, Desclée de Brouwer, 2011, et Ulysse ou la métamorphose, Paris, Kimé, 2020.

5. Nous nous référerons ici à quatre des ouvrages de W. F. Otto (1874-1958). Deux sont accessibles en traduction française : Les Dieux de la Grèce, Paris, Payot, 1981 et L'Esprit de la religion grecque ancienne, "Theophania ", Paris, Agora, Pocket, 2006. Deux sont en langue allemande : Die Gestalt und das Sein, Darmstadt, Wissenschaftliche Buchhandlung, 1955 et Das Wort der Antike, Stuttgart, Klett, 1962.

6. W. F. Otto, Das Wort der Antike, Stuttgart, Klett, 1962, p. 231.

7. Homère, Iliade, VI, 132.

8. M. Heidegger, Nietzsche I, Paris, Gallimard, 1961, p. 363 et 437. Citons par exemple, en modifiant la traduction, ces lignes extraites de la page 437: "Quant à savoir pourquoi l'expérience fondamentale qu'exprime ce mot ne parvint pas à prédominer, ni ne le pouvait, élucider cela reste en dehors de notre tâche présente. "

9. M. Heidegger, Essais et Conférences, Paris, Gallimard, 1966, p. 147.

10. M. Heidegger, Besinnung, Gesamtausgabe, t. 66, Francfort-sur-le-Main, Klostermann, 1997, p. 295.

11. Platon, République, II, $364 \mathrm{~d}$ et Apologie de Socrate, $41 \mathrm{a}$.

12. W. F. Otto, Die Gestalt und das Sein, Darmstadt, Wissenschaftliche Buchhandlung, 1955, p. 315 sq.

13. Homère, Iliade, chant III, 276-280.

14. Homère, Iliade, XIX, 409-10.

15. J. Beaufret, Dialogue avec Heidegger IV, Paris, Éditions de Minuit, 1985, p. 37.

16. W. F. Otto, Das Wort der Antike, Stuttgart, Klett, 1962, p. 334-347.

17. É. Benveniste, Le Vocabulaire des institutions européennes, tome 2, Paris, Éditions de Minuit, 1969 , p. 265 sq. 
18. W. F Otto, Das Wort der Antike, op. cit., p. 339.

19. R. Otto, Le Sacré, Paris, Payot, Petite bibliothèque, 1995.

20. C. Baudelaire, « Bénédiction », Les Fleurs du Mal, La Pléiade, Paris, Gallimard, p. 9.

21. W. F. Otto, Die Gestalt und das Sein, op. cit., p. 151.

22. W. F. Otto, L'Esprit de la religion grecque ancienne, « Theophania ", Paris, Agora Pocket, 2006, p. 104.

23. W. F. Otto, Die Gestalt und das Sein, op. cit., p. 334.

24. W. F. Otto, Die Gestalt und das Sein, op. cit., p. 126.

25. W. F. Otto, Das Wort der Antike, op. cit., p. 39.

26. Ibid. p. 329.

27. Hölderlin, Remarques sur CEdipe, Remarques sur Antigone, Paris, Bibliothèque 10-18, 1965, p. 75.

28. Homère, Odyssée, VII, 201.

29. W. F. Otto, Das Wort der Antike, op. cit., p. 339.

30. Par exemple dans W. F. Otto, Die Gestalt und das Sein, op. cit., p . 22.

31. Goethe, Maximen und Reflexionen, Munich, Hamburger Ausgabe, DTV, tome 12, 1982, p.467.

32. Goethe, ibid., p. 366.

33. Homère, Odyssée, II, 372.

34. Homère, Odyssée, $\mathrm{X}, 300$.

35. Homère, Odyssée, XVI, 160-1.

36. Pindare, Septième Olympique, vers 45-6.

37. C'est Jean Beaufret qui avait autrefois attiré notre attention sur ce terme.

38. Eschyle, Agamemnon, vers 134-5.

39. Ibid., vers 274 .

40. Platon, République, $\mathrm{X}, 596 \mathrm{~d}$-e.

41. K. Axelos, notamment Métamorphoses, Paris, Éditions de Minuit, 1991, p. 135.

42. Homère, Odyssée, VI 232-5 et XXXIII 156-161.

43. R. Char, Recherche de la Base et du Sommet, op.cit., p. 708.

44. R. Char, «Seuil », Le Poème pulvérisé, op. cit., p. 255.

45. M. Heidegger, Nietzsche II, Pfullingen, Neske, 1961, p. 394.

46. F. Nietzsche, Par-delà le bien et le mal, Aphorisme 121, Paris, Éditions 10-18, 1963, p.99. 